\title{
Constitutive Activation of $\beta$-Catenin in Differentiated Osteoclasts Induces Bone Loss in Mice
}

\author{
Xin Sui ${ }^{\mathrm{a}}$ Shijian Deng ${ }^{\mathrm{a}} \quad$ Mengmeng Liu $^{\mathrm{a}}$ Linlin Fan ${ }^{\mathrm{a}}$ Yunfei Wang ${ }^{\mathrm{a}}$ \\ Huaxing $\mathrm{Xu}^{\mathrm{a}}$ Yao Sun ${ }^{\mathrm{b}}$ Anil Kishen ${ }^{\mathrm{c}}$ Qi Zhang ${ }^{\mathrm{a}}$
}

aDepartment of Endodontics, School \& Hospital of Stomatology, Tongji University, Shanghai Engineering Research Center of Tooth Restoration and Regeneration, Shanghai, 'Department of Implantology, School \& Hospital of Stomatology, Tongji University, Shanghai, Engineering Research Center of Tooth Restoration and Regeneration, Shanghai, China, 'Discipline of Endodontics, Faculty of Dentistry, University of Toronto, Toronto, Canada

\section{Key Words}

B-catenin - Wnt/ $\beta$-catenin signalling pathway - Osteoclast - Osteoclastogenesis - Bone homeostasis

\begin{abstract}
Background/Aims: Activation of the Wnt/ $\beta$-catenin signalling pathway has been widely investigated in bone biology and shown to promote bone formation. However, its specific effects on osteoclast differentiation have not been fully elucidated. Our study aimed to identify the role of $\beta$-catenin in osteoclastogenesis and bone homeostasis. Methods: In the present study, exon 3 in the $\beta$-catenin gene (Ctnnb1) allele encoding phosphorylation target serine/ threonine residues was flanked by floxP sequences. We generated mice exhibiting conditional $\beta$-catenin activation (Ctsk-Cre;Ctnnb1flox(exon3)/+ , designated CA- $\beta$-catenin) by crossing

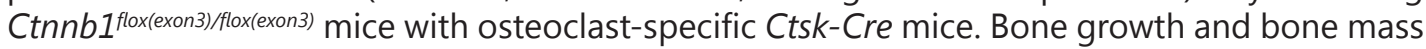
were analysed by micro-computed tomography (micro-CT) and histomorphometry. To further examine osteoclast activity, osteoclasts were induced from bone marrow monocytes (BMMs) isolated from CA- $\beta$-catenin and Control mice in vitro. Osteoclast differentiation was detected by tartrate-resistant acid phosphatase (TRAP) staining, immunofluorescence staining and reverse transcription-quantitative PCR (RT-qPCR) analysis. Results: Growth retardation and low bone mass were observed in CA- $\beta$-catenin mice. Compared to controls, CA- $\beta$-catenin mice had significantly reduced trabecular bone numbers under growth plates as well as thinner cortical bones. Moreover, increased TRAP-positive osteoclasts were observed on the surfaces of trabecular bones and cortical bones in the CA- $\beta$-catenin mice; consistent results were observed in vitro. In the CA- $\beta$-catenin group, excessive numbers of osteoclasts were
\end{abstract}

X. Sui and S. Deng contributed equally to this work.

\begin{tabular}{ll}
\hline Qi Zhang D.D.S, PhD, & Department of Endodontics, School \& Hospital of Stomatology, Tongji University \\
Professor & 399 Middle Yanchang Road, Shanghai, 200072 (China) \\
& Tel. 86-21-66311659; Fax 86-27-66524025; E-Mail qizhang@tongji.edu.cn
\end{tabular}


induced from BMMs, accompanied by the increased expression of osteoclast-associated marker genes. Conclusion: These results indicated that the constitutive activation of $\beta$-catenin in osteoclasts promotes osteoclast formation, resulting in bone loss.

(C) 2018 The Author(s)

Published by S. Karger AG, Basel

\section{Introduction}

$\beta$-catenin, the centre of canonical Wnt signalling, is essential for bone homeostasis [1]. Knockout or activation of $\beta$-catenin at diverse differentiation stages in different bone cells results in distinct phenotypes. For example, $\beta$-catenin knockout in mesenchymal stem cells, osteoblasts and osteocytes results in low bone mass, while the constitutive activation of $\beta$-catenin in these cells increases bone mass. During the early stages of osteoblast differentiation, knocking out $\beta$-catenin in mesenchymal progenitors results in the termination of osteoblast differentiation and abnormal cartilage formation [2]. Thus, $\beta$-catenin is necessary for early osteoblast differentiation, and $\beta$-catenin deficiency promotes mesenchymal stem cells to differentiate into cartilage cells [3]. Knockout of $\beta$-catenin in mature osteoblasts significantly decreases bone mass. Moreover, targeted activation of $\beta$-catenin induces osteopetrosis and increases bone mass [4-6]. With the exception of osteoblasts, $\beta$-catenin knockout in osteocytes results in progressive bone loss in the appendicular and axial skeleton, while constitutive activation of $\beta$-catenin in osteocytes results in high bone mass. These findings confirm the important effects of Wnt/ $\beta$-catenin signalling for maintaining bone mass $[7,8]$. All these findings revealed that $\mathrm{Wnt} / \beta$-catenin signalling exerts essential effects on bone formation and bone mass regulation. However, the effects of Wnt/ $\beta$-catenin signalling on osteoclastogenesis remain unclear.

Osteoclasts, the only bone-resorbing cell type, are derived from the monocyte/ macrophage haematopoietic lineage and play an important role in bone homeostasis [9]. Excessive osteoclastic activity is related to osteoporosis, periodontal disease, osteoarthritis and metastatic cancers [10]. Osteoclast differentiation and activation processes are mainly regulated by receptor activator of nuclear factor- $\kappa B$ ligand (RANKL) signalling [11]. In addition to RANKL, other factors, such as tumour necrosis factor $\alpha$ (TNF $\alpha$ ), interleukins (e.g., IL1 and IL6) and factors released from absorbed bone matrix (e.g., TGFß1 and IGF1), are involved in regulating bone resorption and osteoclastogenesis [12-15]. However, according to previous studies, Wnt/ $\beta$-catenin signalling may exert an indirect inhibitory effect on osteoclast differentiation by promoting the expression of osteoprotegerin $(\mathrm{OPG})[4,5,8,16$, 17].

It has recently been reported that $W n t / \beta$-catenin signalling has a direct effect on the regulation of osteoclastogenesis. $\beta$-catenin deletion in osteoclast progenitors results in osteoporosis in heterozygotes but decreased bone resorption in homozygotes. $\beta$-catenin is necessary for early osteoclast proliferation [18]. Knockout of $\beta$-catenin during the late stages of osteoclast differentiation results in enhanced osteoclast formation and osteopenia with no effects on OPG in mice [18-21]. These findings suggest that inactivation of $\beta$-catenin in osteoclasts results in low bone mass and that $\beta$-catenin plays an important part in osteoclast formation. As mentioned above, activation of $\beta$-catenin is critical for osteoblast lineage cell differentiation during bone formation. However, the effects of stabilized $\beta$-catenin on osteoclast differentiation remain unclear. Therefore, the present work aimed to confirm that the constitutive activation of $\beta$-catenin in osteoclasts plays roles in bone growth, bone mass, and osteoclastogenesis.

The present study investigated the direct functions of $\beta$-catenin stabilization in osteoclasts by crossing Ctnnb1 $1^{\text {flox(exon3)/flox(exon3) }}$ and Ctsk-Cre mice. The generated mice showed a low bone mass phenotype with increased osteoclast formation in vivo and in vitro. Therefore, constitutive activation of $\beta$-catenin may promote osteoclastogenesis, which further implicates the pivotal role of $\beta$-catenin in maintaining bone homeostasis. 


\section{Cellular Physiology Cell Physiol Biochem 2018;48:2091-2102 \begin{tabular}{l|l} 
and Biochemistry Published online: August 9, 2018 & $\begin{array}{l}\text { C } 2018 \text { The Author(s). Published by S. Karger AG, Basel } \\
\text { www.karger.com/cpb }\end{array}$
\end{tabular}}

Sui et al.: Constitutive Activation of B-Catenin in Osteoclasts

\section{Materials and Methods}

\section{Animals}

All experimental procedures were approved by the Animal Welfare Committee of Tongji University (Shanghai, China). To determine the role of stabilized $\beta$-catenin in osteoclasts, Ctnnb1 $1^{\text {flox(exon3)/flox(exon3) }}$ mice (C57BL/6J background, Beijing Biocytogen, China) were crossed with osteoclast-specific Ctsk-Cre mice (C57BL/6J background, Beijing Biocytogen, China) [22, 23]. The Ctsk-Cre mice were knock-in mice and used as heterozygotes. Ctsk-Cre;Ctnnb1 $1^{\text {flox(exon3)/+ }}$ mice (CA- $\beta$-catenin, constitutive activation of $\beta$-catenin) were the target mice, in which exon 3 of the $\beta$-catenin gene (Ctnnb1) in osteoclasts was knocked out to prevent $\beta$-catenin destruction. This alteration permitted the accumulation of cytosolic $\beta$-catenin, which

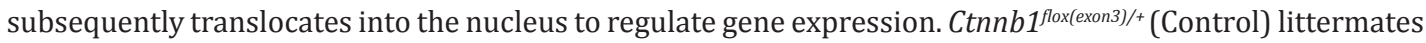
were used as controls. To confirm the site specificity of Ctsk-Cre mice, Rosa26 mT/mG mice were crossed with Ctsk-Cre mice.

\section{Micro-computed tomography (micro-CT) analyses}

Dissected femurs from 4-week-old mice were fixed in 4\% paraformaldehyde for 48 hours $(\mathrm{n}=6$ each group). After fixation, the femurs were scanned and analysed by micro-CT-50 (Scanco Medical, Bassersdorf, Switzerland). Micro-CT analysis included a high-resolution scan of the entire femur bone $(10-\mu \mathrm{m}$ slice increments). For cortical bone analysis, a cylindrical area starting from the mid-diaphysis and extending $1000 \mu \mathrm{m}$ (100 slices) was selected and analysed. For trabecular bone analysis, an area starting from the growth plate in the centre of the metaphysis region with a proximal length of $1000 \mu \mathrm{m}$ (100 slices) was selected. The following quantitative morphometric analyses were determined: bone volume to total volume ratio (BV/TV), mean trabecular thickness (Tb.Th), trabecular separation (Tb.Sp), trabecular number (Tb.N), and mean cortical thickness (Ct.Th).

\section{Bone histology}

After demineralization in 10\% EDTA ( $\mathrm{pH}=7.4)$, femurs were embedded in paraffin and cut into 4- $\mu \mathrm{m}$ thick sections. After deparaffinization and rehydration, the histologic characterization of mouse femurs (n = 5 each group) at 4 weeks was performed by haematoxylin-eosin (H\&E) staining (Biotech Well, China), immunohistochemistry (IHC) staining (UltraSitive S-P detection kit, MXB, China) and tartrate-resistant acid phosphatase (TRAP) staining (Sigma, USA). The number of TRAP-positive osteoclasts per slice in an entire section was counted in five different sections. The mean number was calculated and used for the statistical analyses [24].

\section{In vitro osteoclast differentiation and immunofluorescence staining}

For in vitro osteoclast differentiation analyses, bone marrow cells were isolated from 4-week-old femurs and tibias of Control and CA- $\beta$-catenin mice and cultured for 24 hours with $\alpha$ minimal essential medium ( $\alpha$-MEM) (HyClone, USA) supplemented with 10\% foetal bovine serum (FBS) (Gibco, USA) and $1 \%$ penicillin $(10,000 \mathrm{IU})$-streptomycin $(10,000 \mathrm{mg} / \mathrm{mL})$ (HyClone, USA) as previously described [25, 26]. Non-adherent bone marrow monocytes (BMMs) were harvested and seeded into 24-well plates at a concentration of $5 \times 10^{5}$ cells per well and 6 -well plates at a concentration of $3 \times 10^{6}$ cells per well. The cells were cultured with $50 \mathrm{ng} / \mathrm{mL}$ mouse macrophage colony stimulating factor (M-CSF) (PeproTech, USA) in $\alpha$-MEM (HyClone, USA) containing 10\% FBS for 1-2 days and then stimulated with $50 \mathrm{ng} / \mathrm{mL}$ mouse M-CSF and $100 \mathrm{ng} / \mathrm{mL}$ mouse receptor activator of nuclear factor- $\kappa \mathrm{B}$ ligand (RANKL) (PeproTech, USA) for 4-6 days. All in vitro experiments were normatively repeated at least 4 times.

For TRAP staining, the cells were fixed in $4 \%$ paraformaldehyde and stained using a TRAP staining kit (Sigma, USA). The number of mature osteoclasts per well was counted as multinucleated ( $>3$ nuclei) TRAPpositive cells $[19,27]$. RNA expression was analysed by reverse transcription-quantitative PCR (RT-qPCR) analysis. Protein expression was analysed by Western blotting.

For immunofluorescence staining, the cells were fixed in $4 \%$ paraformaldehyde for 30 minutes, washed 2-3 times with PBS, permeabilized using 0.1\% Triton X-100 in PBS and washed twice with PBS. After blocking, the cells were incubated with anti- $\beta$-catenin (Cell Signalling Technology, USA, 9562, 1:500) and anti-cathepsin K (Proteintech, USA, 11239-1-AP, 1:300) antibodies at $4^{\circ} \mathrm{C}$ overnight or in phalloidin (Sigma, USA, P5282) at $37^{\circ} \mathrm{C}$ for one hour. The cells were then incubated with secondary antibody (Abcam, USA, 
ab150076, 1:1000) if necessary. Finally, the cell nuclei were counterstained with DAPI (Sigma, USA, D9542, 1:1000). Images were obtained by laserscanning confocal microscopy (Leica, Germany).

\section{RT-qPCR analysis}

For the RT-qPCR analysis, total RNA was extracted from the femurs of 4-week-old mice using TRIzol (Takara, Japan). cDNA was synthesized by the reverse transcription of total RNA with a Transcription First Strand cDNA Synthesis Kit (Roche, Switzerland). Gene expression was estimated by real-time qPCR. PCR primers for Trap, Traf6, Mmp9, Nfatc1, Calcr, Oscar, Dcstamp, Tcf, Lef1, Wisp, $c-M y c$ and Gapdh are provided in Table 1. The expression of each gene was normalized to that of Gapdh, and all experiments were repeated 3 times.

\section{Western blotting}

Total protein was extracted from femurs and cells by lysing cells in RIPA lysis buffer (Beyotime, China) supplemented with protease and phosphatase inhibitors. Protein (30 $\mu$ g per lane) was electrophoresed in a $10 \%$ sodium dodecyl sulphate-polyacrylamide (SDS-PAGE) gel and transferred to a polyvinylidene difluoride (PVDF) membrane (Millipore, USA). Membranes were blocked in 5\% BSA for 1 hour and then incubated at $4^{\circ} \mathrm{C}$ overnight with primary antibodies. After three 5 -minute washes with TBS-T $(0.1 \%$ Tween-20), the membranes were incubated with an HRP-conjugated secondary antibody (CWBiotech, China, 1:2000). Target protein was detected using a detection reagent (Immobilon ${ }^{\mathrm{TM}}$ Western, Millipore, USA) and visualized by a chemiluminescent imager (ImageQuant LAS 4000mini). Antibodies targeting the following molecules were used: $\beta$-catenin (Cell Signalling Technology, USA, 9562, 1:1000), non-phospho (active) $\beta$-catenin (Ser33/37/Thr41) (Cell Signalling Technology, USA, 8814, 1:1000), GAPDH (Abways, ab2000, 1:5000) and Lamin B1 (Proteintech, USA, 12987-1-AP, 1:1000). All experiments were normatively repeated at least 3 times.

\section{Statistical analyses}

All data are represented as the mean \pm standard deviation. Statistical analyses were conducted with SPSS statistics 20 (IBM) using a Student's t-test with statistical significance levels of ${ }^{\#} \mathrm{P}<0.05,{ }^{*} \mathrm{P}<0.05$ and $* * \mathrm{P}<0.01$.

\section{Results}

\section{$\beta$-catenin expression pattern in osteoclasts}

The expression of $\beta$-catenin in osteoclasts was detected by immunohistochemistry and immunofluorescence in vivo and in vitro (Fig. 1A-D). Actin ring formation, which is typical of active osteoclasts, was stained with phalloidin (green fluorescence). $\beta$-catenin was located in the nuclei as well as the cytoplasm in osteoclasts. The expression of $\beta$-catenin in osteoclast of CA- $\beta$-catenin mice was much higher than in the control (Fig. 1A-B). To examine $\beta$-catenin expression patterns during the RANKL-mediated osteoclast differentiation process, mRNA expression levels of Trap (as a marker of osteoclast differentiation) and $\beta$-catenin were
Table 1. Murine primer sequences used in RTR. Trap, tartrate-resistant acid phosphatase matrix metalloproteinase-9; Nfatc1, nuclear factor Oscar, osteoclast-associated immunoglobulin-like receptor; Dcstamp, dendrocyte expressed seven Lef-1, lymphoid enhancer binding factor 1 ; Wisp, WNT1 inducible signaling pathway protein; $c-M y c$, phosphate dehydrogenase;

\begin{tabular}{lll}
\hline Genes & \multicolumn{1}{c}{ Forward primer 5' ${ }^{\prime}$ 3' $^{\prime}$} & \multicolumn{1}{c}{ Reverse primer 5'-3' } \\
\hline Trap & CACTCCCACCCTGAGATTTGT & CATCGTCTGCACGGTTCTG \\
Traf6 & AAAGCGAGAGATTCTTTCCCTG & ACTGGGGACAATTCACTAGAGC \\
Mmp9 & TGAGTCCGGCAGACAATCCT & CCACGTGCGGGCAGTAA \\
Nfatc1 & TGAGGCTGGTCTTCCGAGTT & CGCTGGGAACACTCGATAGG \\
Calcr & TCCAACAAGGTGCTTGGGAA & CTTGAACTGCGTCCACTGGC \\
Oscar & GTTTGGGGCTGGCAGGAATGGT & GAGGTGGGGAGCCGGAAATAAGG \\
Dcstamp & TCCTCCATGAACAAACAGTTCCA & AGACGTGGTTTAGGAATGCAGCTC \\
Tcf & CCTCTCTGGCTTCTACTCCCT & CAGCCTGGGTATAGCTGCATGT \\
Lef-1 & TGGCATCCCTCATCCAGCTAT & TGAGGCTTCACGTGCATTAGG \\
Wisp & ACCAATGGCGAGTCCTTC & AGTTCTCATACCGTTGCTCC \\
c-Myc & GCTCTCCATCCTATGTTGCGG & TCCAAGTAACTCGGTCATCATCT \\
Gapdh & GGGAAGCCCATCACCATCTT & GCCTCACCCCATTTGATGTT \\
\hline
\end{tabular}


Fig. 1. Localization of $\beta$-catenin in osteoclasts. (AD) Immunohistochemistry on bone tissue and immunofluorescence on cultured osteoclasts demonstrated the presence of $\beta$-catenin in osteoclasts in vivo (black arrows) and in vitro. (E) RT-qPCR expression analysis of $\beta$-catenin and Trap in osteoclasts induced from wild type murine BMMs in the presence of M-CSF and RANKL. (F$\mathrm{H})$ Western blot analysis of $\beta$-catenin and non-phospho (active) $\beta$-catenin (Ser33/37/Thr41) in osteoclasts derived from wild type mice during the differentiation period. Scale bar $=50 \mu \mathrm{m}$. BMMs: bone marrow monocytes. Trap mRNA levels on days 1,3 , and 6 were compared to Trap mRNA levels on days 0,1 , and 3 , respectively. $\beta$-catenin mRNA levels on days 1,3 , and 6 were compared to $\beta$-catenin mRNA levels on days 0,1 , and 3 , respectively. All experiments were normatively repeated at least 3 times. ${ }^{\#} \mathrm{P}<0.05$, ${ }^{*} \mathrm{P}<0.05$ and $* * \mathrm{P}<0.01$.
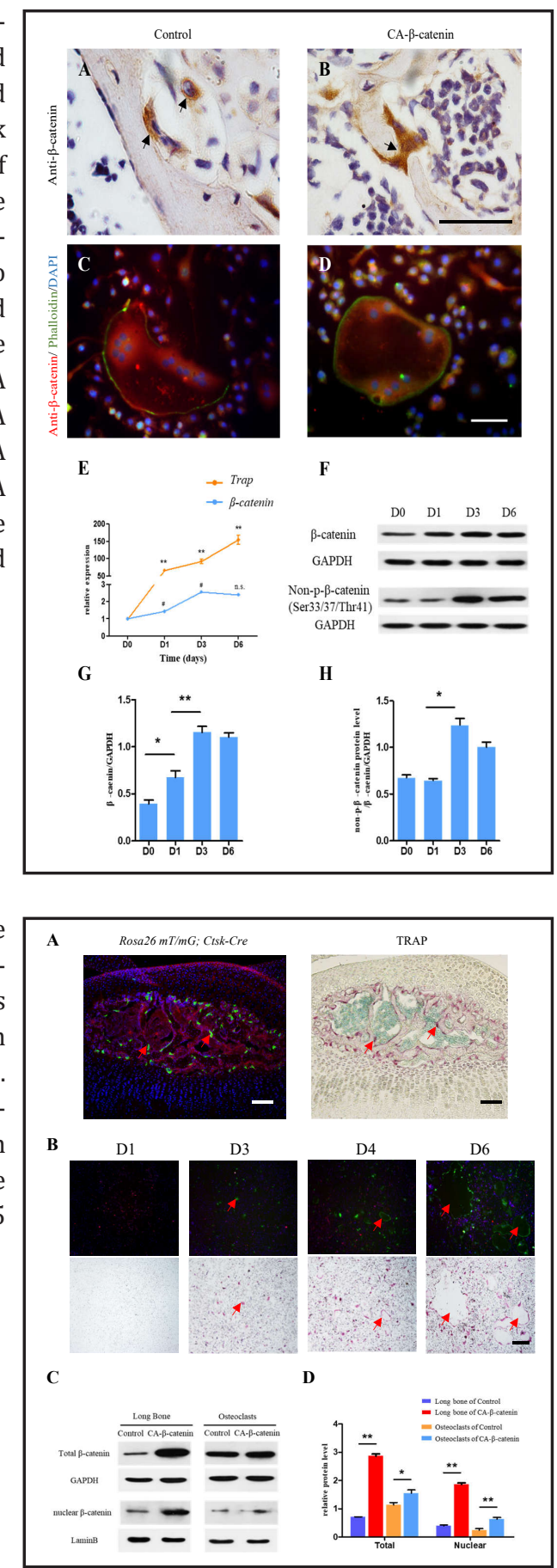

Fig. 2. Ctsk-Cre site-specific expression patterns. (A) Cre expression pattern in the bones of Rosa $26 \mathrm{mT} / \mathrm{mG}$; CtskCre mice. Green fluorescence marks cells that express Cre protein. (B) Cre expression patterns on days 1-6 in osteoclasts induced from Rosa $26 \mathrm{mT} / \mathrm{mG}$; Ctsk-Cre mice. TRAP staining shows mature osteoclasts (red arrows). (CD) Western blot analysis of total and nuclear $\beta$-catenin in long bones in vivo and induced osteoclasts in vitro. Scale bar $=100 \mu \mathrm{m}$. BMMs, bone marrow monocytes. ${ }^{*} \mathrm{P}<0.05$ and ${ }^{* *} \mathrm{P}<0.01$.

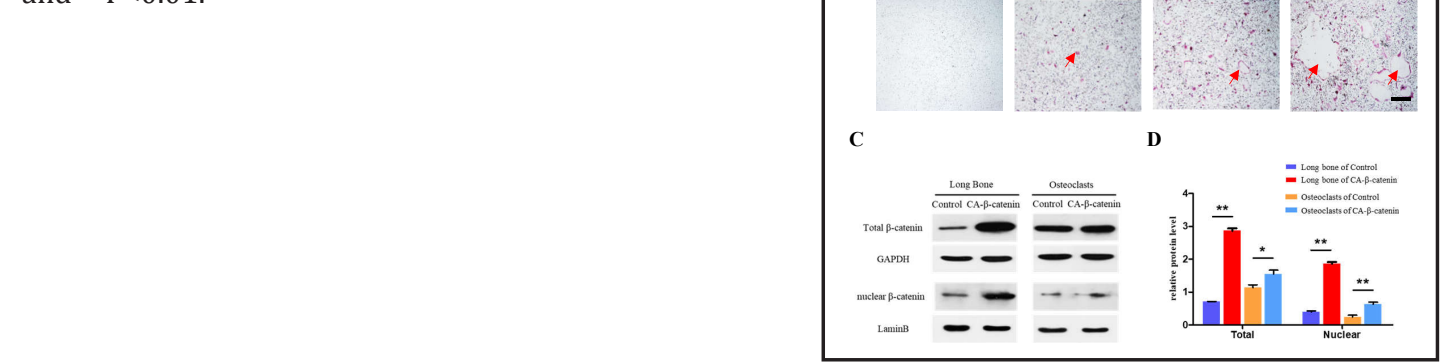

detected under normal conditions (Fig. 1E). Trap and $\beta$-catenin expression levels increased gradually for 1-3 days in the presence of M-CSF and RANKL during osteoclastogenesis. Then, during the osteoclast maturation stage (days 4-6), the high expression levels of $\beta$-catenin were slightly downregulated. Consistently, Western blotting results showed that the expression of non-phosphorylated $\beta$-catenin increased during osteoclast differentiation (Fig. 1F-H). Non-phosphorylated $\beta$-catenin, which is translocated to the nucleus when cells receive canonical Wnt signalling, is the stabilized form of $\beta$-catenin $[28,29]$. These findings implied that osteoclasts may be regulated by canonical Wnt signalling in osteoclastogenesis. Ctsk-Cre site-specific expression was demonstrated in osteoclasts using reporter mice 
Fig. 3. Growth retardation and low bone mass in CA- $\beta$ catenin mice. (A) CA- $\beta$-catenin mice showed smaller and shorter statures at 4 weeks of age. (B) Body weights of CA- $\beta$ catenin and Control mice at 1-6 weeks postnatal. (C) Threedimensional reconstruction and coronal sections of Control and CA- $\beta$-catenin mice (4 weeks old) determined via microCT analysis. (D) Reconstructed trabecular bone of distal femurs and cortical bone of middle femurs in Control and CA- $\beta$-catenin mice (cross-section level). (E) Quantitative morphometric analyses based on micro-CT. BV/TV, bone volume to total volume; Tb.Th, ratio mean trabecular thickness; Tb.Sp, trabecular separation; Tb.N, trabecular number; and Ct.Th, mean cortical thickness. Scale bar $=5$ mm. $* \mathrm{P}<0.05$ and ${ }^{* *} \mathrm{P}<0.01$.

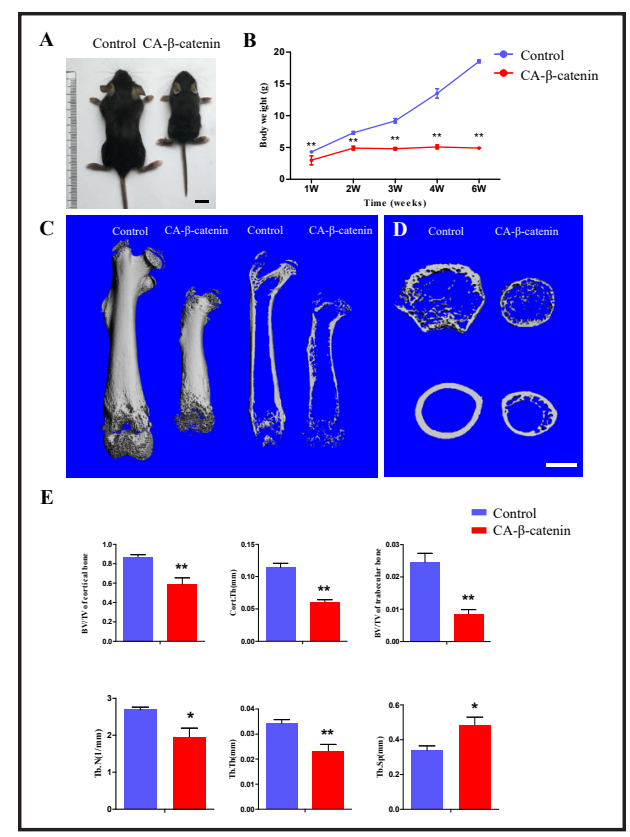

(Fig. 2A-B). Additionally, total and nuclear $\beta$-catenin expression increased in both long bones and in induced osteoclasts in vitro after $\beta$-catenin activation, indicating that $\beta$-catenin signalling increased in osteoclasts of CA- $\beta$-catenin mice (Fig. 2C-D).

Developmental retardation and low bone mass in $C A-\beta$-catenin mice

After demonstrating $\beta$-catenin expression, we generated CA- $\beta$-catenin mice by intercrossing Ctnnb1 $1^{\text {flox(exon3)/ }}$ flox(exon3) mice with Ctsk-Cre mice to achieve
Table 2. Statistics of micro-CT analysis. ${ }^{a} \mathrm{p} \leq 0.05$, ${ }^{b} \mathrm{p} \leq 0.01$

\begin{tabular}{lccc}
\hline \multicolumn{3}{c}{ Histomorphometric analysis (mean \pm SD) } & \\
\hline & Control & CA- $\beta$-catenin & P value \\
BV/TV of cortical bone (\%) & $0.870 \pm 0.0537$ & $0.587 \pm 0.1641$ & $0.002^{\mathrm{b}}$ \\
BV/TV of trabecular bone (\%) & $0.025 \pm 0.0067$ & $0.0086 \pm 0.0031$ & $0.001^{\mathrm{b}}$ \\
Tb.Th $(\mathrm{mm})$ & $0.034 \pm 0.0036$ & $0.023 \pm 0.0069$ & $0.006^{\mathrm{b}}$ \\
Tb. Sp $(\mathrm{mm})$ & $0.339 \pm 0.0622$ & $0.485 \pm 0.1078$ & $0.016^{\mathrm{a}}$ \\
Tb. N $(1 / \mathrm{mm})$ & $2.692 \pm 0.1606$ & $1.957 \pm 0.5736$ & $0.013^{\mathrm{a}}$ \\
Ct. Th $(\mathrm{mm})$ & $0.114 \pm 0.01511$ & $0.060 \pm 0.0104$ & $0.001^{\mathrm{b}}$ \\
\hline
\end{tabular}

$\beta$-catenin stabilization in osteoclasts. CA- $\beta$-catenin mice displayed shorter and smaller body statures than their Control littermates (Fig. 3A). There were no overt gender differences for this phenotype. Moreover, the weight of $C A-\beta$-catenin mice was also lower than that of Control mice (Fig. 3B). Almost all the CA- $\beta$-catenin mice died at 6-7 weeks of age.

According to the micro-CT results, $C A-\beta$-catenin mice exhibited an obvious osteoporosis phenotype. Both three-dimensional reconstruction and coronal sections showed that the CA$\beta$-catenin mice had shorter femurs and thinner cortical bones than the Control mice (Fig. 3C). At the cross-section level, these phenotypes were further demonstrated by reconstruction of the trabecular bone of the distal femur and the cortical bone of the middle femur (Fig. 3D). Quantitative morphometric analyses were consistent with low bone mass phenotypes, including the BV/TVs of cortical and trabecular bones, Tb.Th, Tb.Sp, Tb.N, and Ct.Th (Fig. 3E). Detailed statistics are shown in Table 2. Overall, these findings suggest that $\beta$-catenin stabilization in osteoclasts induces shorter stature and lower bone mass.

Histological loss of bone mass and increased osteoclasts in CA- $\beta$-catenin mice

In accordance with the micro-CT results, the histological staining analysis of CA- $\beta$ catenin mouse femurs showed severe loss of bone mass. There was a significant reduction in both the number of trabecular bones under growth plates and the thickness of the cortical bones (Fig. 4A). Based on histochemical staining, bone mass loss (CA- $\beta$-catenin, 4 weeks old) was characterized by an increased number of TRAP-positive osteoclasts compared 
with the Control group (Fig. 4B). Quantitative statistical analysis of osteoclast numbers showed a 1.5-fold increase in TRAPpositive osteoclasts in CA- $\beta$ catenin mice (Fig. 4C). The expression levels of osteoclastspecific genes were detected and compared to assess osteoclast functions in vivo. The expression levels of Trap, Nfatc1, Mmp9, Traf6, and Dcstamp were higher in the femurs from $C A-\beta$-catenin mice (Fig. 4D). The expression levels of $W n t / \beta$-catenin signalling-related genes, such as Tcf, Lef-1, Wisp and $c-M y c$, were significantly up-regulated in CA- $\beta$-catenin mice. These results suggested that increased osteoclast numbers due to activation of $\mathrm{Wnt} / \beta$-catenin signalling play an important role in bone mass loss in CA- $\beta$ catenin mice.

\section{Enhanced osteoclastogenesis}

in $C A-\beta$-catenin mice in vitro

To evaluate the function of $\beta$-catenin in the regulation of osteoclastogenesis, osteoclasts were induced from BMMs isolated from CA- $\beta$-catenin and Control mice in vitro. The number of osteoclasts was detected by TRAP staining (Fig. 5A, B and G), CTSK expression (observed by immunofluorescence staining) (Fig. 5C, D and H), actin ring formation (phalloidin labelling) (Fig. 5E, F and I) and the expression of osteoclast-related genes (Fig. 5J). Osteoclast differentiation and maturation were upregulated in CA- $\beta$ catenin mice, displayed greater numbers of TRAP-positive cells, CTSK-positive cells as well as more osteoclasts with actin ring (Fig. 5A-I). Additionally, the expression of osteoclast-related markers and Wnt/ $\beta$ catenin signalling-related main genes increased, including Trap, Nfatc1, Mmp9, Traf6, Ctr, Oscar, Dcstamp, Tcf, Lef-1, Wisp and c-Myc, which showed that osteoclast differentiation was enhanced in CA- $\beta$-catenin mice (Fig. 5J). Therefore, these data demonstrated that constitutively activated $\beta$-catenin upregulates the osteoclastogenesis process in vitro.
Fig. 4. Histological and functional changes in CA$\beta$-catenin mice. (A) $\mathrm{H} \& \mathrm{E}$ staining analysis of distal femurs (Control and CA$\beta$-catenin mice, 4 weeks old). High magnification of trabecular bone and cortical bone sections. (B) Increased TRAP-positive osteoclasts in trabecular bone under growth plates and cortical bone (CA- $\beta$-catenin, 4 weeks old) compared to Control littermates. (C) Quantitative statistics for TRAP-positive osteoclasts in each group. (D) mRNA expression levels of $\mathrm{Wnt} / \beta$-catenin signalling molecules and osteoclastspecific markers in CA- $\beta$ catenin mice. Scale bar $=200$ $\mu \mathrm{m},{ }^{*} \mathrm{P}<0.05$ and ${ }^{* *} \mathrm{P}<0.01$.

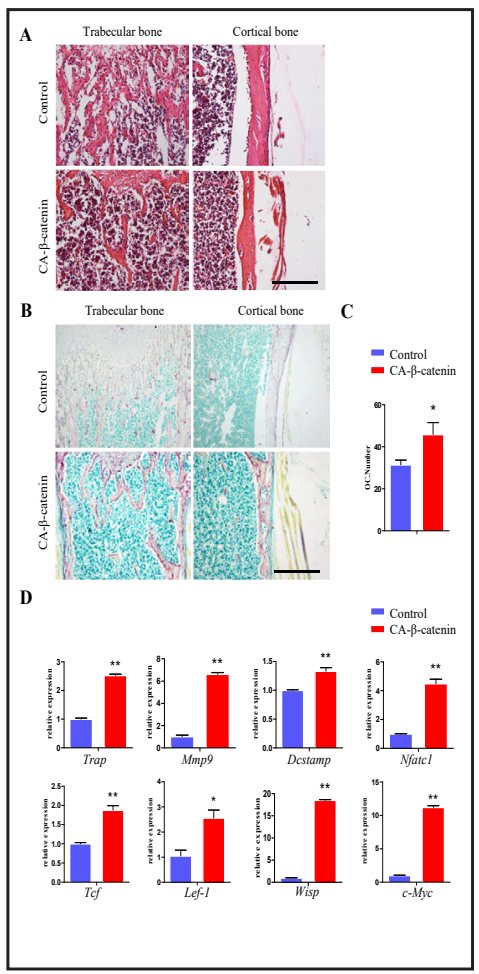

Fig. 5. In vitro osteoclastogenesis of BMMs derived from Control and CA- $\beta$-catenin mice. The degree of differentiation was tested by TRAP staining (A-B), CTSK (C-D), and actin ring formation assays (E-F). (GI) Quantitative statistics for TRAP-positive cells, CTSK-positive cells, and osteoclasts with actin rings. (J) The expression levels in cultured osteoclasts. BMMs, bone marrow monocytes. Scale bar $=250 \mu \mathrm{m}$. All in vitro experiments were normatively repeated at least 4 times. ${ }^{*} \mathrm{P}<0.05$ and $* * \mathrm{P}<0.01$.

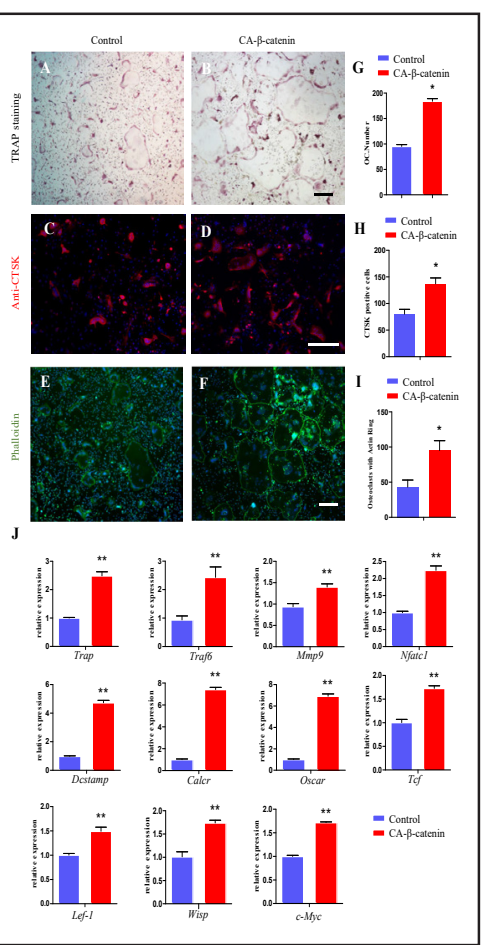


Expression of $\beta$-catenin in other organs in $C A-\beta$-catenin mice

It has also been reported that CTSK can be expressed in other organs. Therefore, the expression of $\beta$-catenin was studied in several organs, including heart, liver, lung, kidney, brain and testis (Fig. 6). The results showed that the expression of $\beta$-catenin was increased in liver in $C A-\beta$-catenin mice.

\section{Discussion}

The inhibition of osteoclast differentiation is an effective strategy to treat osteoporosis [30]. Thus, it is important to understand the mechanism underlying osteoclast formation. The Wnt/ $\beta$-catenin pathway is essential for bone mass maintenance via the regulation of bone-forming osteoblasts [31]. However, the specific role of the Wnt/ $\beta$-catenin pathway in osteoclast differentiation remains unclear. Hence, it is necessary to study the potential effects of the Wnt/ $\beta$-catenin pathway on osteoclast-mediated bone resorption to provide a foundation for the treatment of osteoporosis. In this study, an in vivo mouse model was utilized to specifically activate $\beta$-catenin in osteoclasts. The results showed low bone mass in mice associated with enhanced osteoclast activity.

The specificity and validity of the mouse model is the basic principle. Theoretically, CTSK was predominantly thought to be unique to osteoclasts; however, it was also found in other organs [32, 33]. To confirm the specific expression of Ctsk-Cre mice, a dual-fluorescence gene-expression-reporter system and Western blotting analysis were applied and directly verified the site-specific expression pattern in osteoclasts. Moreover, the expression of $\beta$-catenin was also observed in several organs. According to our observations, though the expression of $\beta$-catenin in the liver increased, no obvious changes in the tissue structure and cell morphology were found. However, it does not rule out changes in liver function, which may result in systemic effects affecting bone. The possibility of systemic effects is a limitation of the present study.

In the present study, low bone mass was associated with enhanced osteoclast formation under $\beta$-catenin stabilization. $\beta$-catenin has been reported to upregulate RANKL through sclerostin to induce osteoclastogenesis processes [34]. Recently, c-Myc, a vital target of $\beta$-catenin, was demonstrated to mediate RANK/RANKL signalling in an NFATc1-dependent or NFATc1-independent manner. The c-Myc/ERR $\alpha$ pathway exerts significant effects on osteoclast energy metabolism, which is essential for bone resorption [35-37]. Consistently, the expression levels of $c-M y c$ and molecules downstream of RANK/RANKL signalling increased in our study. Thus, $\beta$-catenin may regulate osteoclast differentiation via c-Myc and downstream signalling pathways. Moreover, the Wnt/ $\beta$-catenin pathway acts as an upstream activator of the phosphatidylinositol 3-kinase/serine/threonine kinase (protein kinase B)/ 
mammalian target of rapamycin (PI3K/Akt/mTOR) pathway, which is a critical RANK/ RANKL downstream signalling pathway [38-40]. However, the potential crosstalk between $\mathrm{Wnt} / \beta$-catenin and other signalling pathways in osteoclastogenesis requires further studies.

However, there are several findings regarding the effects of the $\mathrm{Wnt} / \beta$-catenin signalling pathway on osteoclastogenesis. Loss-of-function or gain-of-function mutations in Lrp5, a Wnt/ $\beta$-catenin signalling receptor, do not alter bone resorption [41]. The deletion of Sost or Dkk1, genes encoding inhibitors of the Wnt/ $\beta$-catenin pathway, does not change osteoclast numbers [42-44]. Furthermore, studies have shown that $\beta$-catenin negatively regulates osteoclasts independent of osteoblasts [19-21]. Interestingly, our present work demonstrated that the activation of $\beta$-catenin in osteoclasts increased osteoclastic activity. We propose two possible reasons for this difference in osteoclastogenesis. On one hand, this contrast may be related to differences between in vivo and in vitro studies. In vivo studies may reflect a complex but real microenvironment, resulting in an integrated phenotype. On the other hand, the manipulation of GSK3 $\beta$ likely alters a variety of signalling pathways, which may explain these contradictory results. GSK3 $\beta$ is a component of many pathways, such as the Wnt and Hedgehog signalling pathways $[45,46]$. GSK3 $\beta$ is also a key downstream effector of Akt pathway, which is required for osteoclast differentiation [47]. Moreover, mice overexpressing GSK3 $\beta$ driven by the TRAP promoter display osteopetrosis due to decreased osteoclast numbers [48].

Wei and colleagues [18] reported that Ctsk-bCA mice exhibited an increased BV/TV ratio and decreased bone resorption by some statistical analysis, which was the opposite of our results. However, its detailed characterization, such as micro-CT analysis, histology analysis, and in vitro study, was quite limited. In this study, we validated the mouse model and performed morphological analyses on in vivo and in vitro osteoclast cultures. These data are presented objectively and systematically. Knockout of $\beta$-catenin in CTSK-expressing cells upregulates osteoclast-related gene expression and results in osteoporosis phenotypes [19]. Knockout or activation of $\beta$-catenin in bone-related cells, such as mesenchyme stem cells or osteoclast precursors, has similar phenotypes in bone $[2,18]$. It is speculated that $\beta$-catenin exerts a biphasic regulation in osteoclastogenesis and leads to similar phenotypes. These similar phenotypes may be related to the complex and multidirectional effects of $\beta$-catenin, although the detailed mechanisms require further studies.

Unexpectedly, CA- $\beta$-catenin mice died early at 6-7 weeks of age. It is speculated that the early death of CA- $\beta$-catenin mice is partly due to severe osteoporosis and bone dysplasia, causing the abnormal development of other organs. On one hand, severe skeletal dysplasia leads to death. $\beta$-catenin is an important molecule and its dysfunction may lead to severe phenotypes in bone, which may be responsible for the death. Many studies have reported that deletion or constitutive activation of $\beta$-catenin in Cre-expression cells, such as Prx1Cre, Osx1-Cre, Col1 1 1-Cre, OCN-Cre, Dmp1-Cre, Tie2-Cre and so on, causes early death in mice $[2,4,5,7,8,49,50]$. On the other hand, bone dysplasia, such as in the hypoplastic thorax, short limbs and ribs, causes damages to other organs and results in death [51,52]. The exact reasons and mechanisms for this still require further investigation.

Our research found that $\beta$-catenin stabilization in osteoclasts might promote osteoclastogenesis. These findings are helpful for understanding the complex role of the Wnt/ $\beta$-catenin signalling pathway in osteoclast differentiation and bone resorption. Moreover, this study may assist with the treatment of osteoporosis and other osteopenia diseases.

\section{Acknowledgements}

This work was supported by the National Natural Science Foundation of China (grant numbers 81570966 and 81371141) and the Specialized Research Fund for the Doctoral Program of Higher Education (grant number 20130072110020).

Xin Sui, Shijian Deng and Qi Zhang contributed to the conception; design; and data 


\section{Cellular Physiology Cell Physiol Biochem 2018;48:2091-2102 \begin{tabular}{l|l|l} 
DOI: 10.1159/000492549 & and Biochemistry Published online: August 9, 2018 & $\begin{array}{l}\text { O } 2018 \text { The Author(s). Published by S. Karger AG, Basel } \\
\text { www.karger.com/cpb }\end{array}$ \\
\cline { 2 - 3 } &
\end{tabular}}

Sui et al.: Constitutive Activation of B-Catenin in Osteoclasts

acquisition, analysis, and interpretation, as well as drafting and critically revising the manuscript. Mengmeng Liu, Linlin Fan, Yunfei Wang and Huaxing Xu contributed to the conception, design, and data acquisition. Yao Sun and Anil Kishen contributed to the conception and design and critically revised the manuscript.

\section{Disclosure Statement}

All the authors declare that they have no conflict of interests related to this report.

\section{References}

-1 Nusse R, Clevers H: Wnt/beta-Catenin Signaling, Disease, and Emerging Therapeutic Modalities. Cell 2017;169:985-999.

-2 Hill TP, Spater D, Taketo MM, Birchmeier W, Hartmann C: Canonical Wnt/beta-catenin signaling prevents osteoblasts from differentiating into chondrocytes. Dev Cell 2005;8:727-738.

-3 Hu H, Hilton MJ, Tu X, Yu K, Ornitz DM, Long F: Sequential roles of Hedgehog and Wnt signaling in osteoblast development. Development 2005;132:49-60.

4 Glass DA, 2nd, Bialek P, Ahn JD, Starbuck M, Patel MS, Clevers H, Taketo MM, Long F, McMahon AP, Lang RA, Karsenty G: Canonical Wnt signaling in differentiated osteoblasts controls osteoclast differentiation. Dev Cell 2005;8:751-764.

-5 Holmen SL, Zylstra CR, Mukherjee A, Sigler RE, Faugere MC, Bouxsein ML, Deng L, Clemens TL, Williams BO: Essential role of beta-catenin in postnatal bone acquisition. J Biol Chem 2005;280:21162-21168.

6 Song L, Liu M, Ono N, Bringhurst FR, Kronenberg HM, Guo J: Loss of wnt/beta-catenin signaling causes cell fate shift of preosteoblasts from osteoblasts to adipocytes. J Bone Miner Res 2012;27:2344-2358.

7 Kramer I, Halleux C, Keller H, Pegurri M, Gooi JH, Weber PB, Feng JQ Bonewald LF, Kneissel M: Osteocyte Wnt/beta-catenin signaling is required for normal bone homeostasis. Mol Cell Biol 2010;30:3071-3085.

8 Chen S, Feng J, Bao Q Li A, Zhang B, Shen Y, Zhao Y, Guo Q Jing J, Lin S, Zong Z: Adverse Effects of Osteocytic Constitutive Activation of ss-Catenin on Bone Strength and Bone Growth. J Bone Miner Res 2015;30:11841194.

9 Novack DV, Teitelbaum SL: The osteoclast: friend or foe? Annu Rev Pathol 2008;3:457-484.

10 Takayanagi H: Osteoimmunology: shared mechanisms and crosstalk between the immune and bone systems. Nat Rev Immunol 2007;7:292-304.

-11 Liu W, Zhang X: Receptor activator of nuclear factor-kappaB ligand (RANKL)/RANK/osteoprotegerin system in bone and other tissues (review). Mol Med Rep 2015;11:3212-3218.

12 Sims NA, Martin TJ: Coupling the activities of bone formation and resorption: a multitude of signals within the basic multicellular unit. Bonekey Rep 2014;3:481.

13 Lam J, Takeshita S, Barker JE, Kanagawa O, Ross FP, Teitelbaum SL: TNF-alpha induces osteoclastogenesis by direct stimulation of macrophages exposed to permissive levels of RANK ligand. J Clin Invest 2000;106:1481-1488.

-14 Santos Savio A, Machado Diaz AC, Chico Capote A, Miranda Navarro J, Rodriguez Alvarez Y, Bringas Perez R, Estevez del Toro M, Guillen Nieto GE: Differential expression of pro-inflammatory cytokines IL-15Ralpha, IL-15, IL-6 and TNFalpha in synovial fluid from rheumatoid arthritis patients. BMC Musculoskelet Disord 2015;16:51.

15 Wu Q, Zhou X, Huang D, Ji Y, Kang F: IL-6 Enhances Osteocyte-Mediated Osteoclastogenesis by Promoting JAK2 and RANKL Activity in vitro. Cell Physiol Biochem 2017;41:1360-1369.

-16 Day TF, Guo X, Garrett-Beal L, Yang Y: Wnt/beta-catenin signaling in mesenchymal progenitors controls osteoblast and chondrocyte differentiation during vertebrate skeletogenesis. Dev Cell 2005;8:739-750.

17 Boyle WJ, Simonet WS, Lacey DL: Osteoclast differentiation and activation. Nature 2003;423:337-342.

18 Wei W, Zeve D, Suh JM, Wang X, Du Y, Zerwekh JE, Dechow PC, Graff JM, Wan Y: Biphasic and dosagedependent regulation of osteoclastogenesis by beta-catenin. Mol Cell Biol 2011;31:4706-4719. 


\section{Cellular Physiology Cell Physiol Biochem 2018;48:2091-2102 \begin{tabular}{ll|l} 
and Biochemistry Published online: August 9, 2018 & $\begin{array}{l}\text { (c) } 2018 \text { The Author(s). Published by S. Karger AG, Basel } \\
\text { www.karger.com/cpb }\end{array}$
\end{tabular}}

Sui et al.: Constitutive Activation of B-Catenin in Osteoclasts

19 Ruiz P, Martin-Millan M, Gonzalez-Martin MC, Almeida M, Gonzalez-Macias J, Ros MA: CathepsinKCre mediated deletion of betacatenin results in dramatic loss of bone mass by targeting both osteoclasts and osteoblastic cells. Sci Rep 2016;6:36201.

-20 Otero K, Shinohara M, Zhao H, Cella M, Gilfillan S, Colucci A, Faccio R, Ross FP, Teitelbaum SL, Takayanagi H, Colonna M: TREM2 and beta-catenin regulate bone homeostasis by controlling the rate of osteoclastogenesis. J Immunol 2012;188:2612-2621.

-21 Albers J, Keller J, Baranowsky A, Beil FT, Catala-Lehnen P, Schulze J, Amling M, Schinke T: Canonical Wnt signaling inhibits osteoclastogenesis independent of osteoprotegerin. J Cell Biol 2013;200:537-549.

-22 Harada N, Tamai Y, Ishikawa T, Sauer B, Takaku K, Oshima M, Taketo MM: Intestinal polyposis in mice with a dominant stable mutation of the beta-catenin gene. EMBO J 1999;18:5931-5942.

23 Nakamura T, Imai Y, Matsumoto T, Sato S, Takeuchi K, Igarashi K, Harada Y, Azuma Y, Krust A, Yamamoto Y, Nishina H, Takeda S, Takayanagi H, Metzger D, Kanno J, Takaoka K, Martin TJ, Chambon P, Kato S: Estrogen prevents bone loss via estrogen receptor alpha and induction of Fas ligand in osteoclasts. Cell 2007;130:811-823.

24 Liu Y, Yang R, Shi S: Systemic infusion of mesenchymal stem cells improves cell-based bone regeneration via upregulation of regulatory T cells. Tissue Eng Part A 2015;21:498-509.

25 Takeshita S, Kaji K, Kudo A: Identification and characterization of the new osteoclast progenitor with macrophage phenotypes being able to differentiate into mature osteoclasts. J Bone Miner Res 2000;15:1477-1488.

-26 Tang L, Yin Y, Liu J, Li Z, Lu X: MiR-124 Attenuates Osteoclastogenic Differentiation of Bone Marrow Monocytes Via Targeting Rab27a. Cell Physiol Biochem 2017;43:1663-1672.

$>27$ Kong L, Wang B, Yang X, Guo H, Zhang K, Zhu Z, Liu J, Hao D: Picrasidine I from Picrasma Quassioides Suppresses Osteoclastogenesis via Inhibition of RANKL Induced Signaling Pathways and Attenuation of ROS Production. Cell Physiol Biochem 2017;43:1425-1435.

28 Lu L, Gao Y, Zhang Z, Cao Q Zhang X, Zou J, Cao Y: Kdm2a/b Lysine Demethylases Regulate Canonical Wnt Signaling by Modulating the Stability of Nuclear beta-Catenin. Dev Cell 2015;33:660-674.

29 Valenta T, Hausmann G, Basler K: The many faces and functions of beta-catenin. EMBO J 2012;31:27142736.

-30 Zaidi M: Skeletal remodeling in health and disease. Nat Med 2007;13:791-801.

31 Lerner UH, Ohlsson C: The WNT system: background and its role in bone. J Intern Med 2015;277:630-649.

32 Chiu WS, McManus JF, Notini AJ, Cassady AI, Zajac JD, Davey RA: Transgenic mice that express Cre recombinase in osteoclasts. Genesis 2004;39:178-185.

33 Winkeler CL, Kladney RD, Maggi LB, Jr., Weber JD: Cathepsin K-Cre causes unexpected germline deletion of genes in mice. PLoS One 2012;7:e42005.

-34 Wijenayaka AR, Kogawa M, Lim HP, Bonewald LF, Findlay DM, Atkins GJ: Sclerostin stimulates osteocyte support of osteoclast activity by a RANKL-dependent pathway. PLoS One 2011;6:e25900.

-35 Bae S, Lee MJ, Mun SH, Giannopoulou EG, Yong-Gonzalez V, Cross JR, Murata K, Giguere V, van der Meulen M, Park-Min KH: MYC-dependent oxidative metabolism regulates osteoclastogenesis via nuclear receptor ERRalpha. J Clin Invest 2017;127:2555-2568.

-36 Park-Min KH, Lim E, Lee MJ, Park SH, Giannopoulou E, Yarilina A, van der Meulen M, Zhao B, Smithers N, Witherington J, Lee K, Tak PP, Prinjha RK, Ivashkiv LB: Inhibition of osteoclastogenesis and inflammatory bone resorption by targeting BET proteins and epigenetic regulation. Nat Commun 2014;5:5418.

-37 Battaglino R, Kim D, Fu J, Vaage B, Fu XY, Stashenko P: c-myc is required for osteoclast differentiation. J Bone Miner Res 2002;17:763-773.

38 Vallee A, Lecarpentier Y, Guillevin R, Vallee JN: Interactions between TGF-beta1, canonical WNT/betacatenin pathway and PPAR gamma in radiation-induced fibrosis. Oncotarget 2017;8:90579-90604.

39 Chen J, Alberts I, Li X: Dysregulation of the IGF-I/PI3K/AKT/mTOR signaling pathway in autism spectrum disorders. Int J Dev Neurosci 2014;35:35-41.

-40 Huang J, Nguyen-McCarty M, Hexner EO, Danet-Desnoyers G, Klein PS: Maintenance of hematopoietic stem cells through regulation of Wnt and mTOR pathways. Nat Med 2012;18:1778-1785. 


\section{Cellular Physiology Cell Physiol Biochem 2018;48:2091-2102

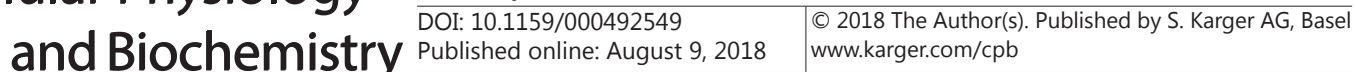

41 Little RD, Carulli JP, Del Mastro RG, Dupuis J, Osborne M, Folz C, Manning SP, Swain PM, Zhao SC, Eustace B, Lappe MM, Spitzer L, Zweier S, Braunschweiger K, Benchekroun Y, Hu X, Adair R, Chee L, FitzGerald MG, Tulig C, Caruso A, et al.: A mutation in the LDL receptor-related protein 5 gene results in the autosomal dominant high-bone-mass trait. Am J Hum Genet 2002;70:11-19.

42 Li X, Ominsky MS, Niu QT, Sun N, Daugherty B, D’Agostin D, Kurahara C, Gao Y, Cao J, Gong J, Asuncion F, Barrero M, Warmington K, Dwyer D, Stolina M, Morony S, Sarosi I, Kostenuik PJ, Lacey DL, Simonet WS, et al.: Targeted deletion of the sclerostin gene in mice results in increased bone formation and bone strength. J Bone Miner Res 2008;23:860-869.

-43 Lin C, Jiang X, Dai Z, Guo X, Weng T, Wang J, Li Y, Feng G, Gao X, He L: Sclerostin mediates bone response to mechanical unloading through antagonizing Wnt/beta-catenin signaling. J Bone Miner Res 2009;24:16511661.

44 Mukhopadhyay M, Shtrom S, Rodriguez-Esteban C, Chen L, Tsukui T, Gomer L, Dorward DW, Glinka A, Grinberg A, Huang SP, Niehrs C, Izpisua Belmonte JC, Westphal H: Dickkopf1 is required for embryonic head induction and limb morphogenesis in the mouse. Dev Cell 2001;1:423-434.

-45 Doble BW, Woodgett JR: Role of glycogen synthase kinase-3 in cell fate and epithelial-mesenchymal transitions. Cells Tissues Organs 2007;185:73-84.

46 Wu D, Pan W: GSK3: a multifaceted kinase in Wnt signaling. Trends Biochem Sci 2010;35:161-168.

47 Liang J, Slingerland JM: Multiple roles of the PI3K/PKB (Akt) pathway in cell cycle progression. Cell Cycle 2003;2:339-345.

48 Jang HD, Shin JH, Park DR, Hong JH, Yoon K, Ko R, Ko CY, Kim HS, Jeong D, Kim N, Lee SY: Inactivation of glycogen synthase kinase-3beta is required for osteoclast differentiation. J Biol Chem 2011;286:3904339050.

49 Kode A, Manavalan JS, Mosialou I, Bhagat G, Rathinam CV, Luo N, Khiabanian H, Lee A, Murty VV, Friedman R, Brum A, Park D, Galili N, Mukherjee S, Teruya-Feldstein J, Raza A, Rabadan R, Berman E, Kousteni S: Leukaemogenesis induced by an activating beta-catenin mutation in osteoblasts. Nature 2014;506:240244.

50 Rodda SJ, McMahon AP: Distinct roles for Hedgehog and canonical Wnt signaling in specification, differentiation and maintenance of osteoblast progenitors. Development 2006;133:3231-3244.

51 Chowdhury D, Williams KB, Chidekel A, Pizarro C, Preedy C, Young M, Hendrickson C, Robinson DL, Kreiger PA, Puffenberger EG, Strauss KA: Management of Congenital Heart Disease Associated with Ellis-van Creveld Short-rib Thoracic Dysplasia. J Pediatr 2017;191:145-151.

52 Rauch F, Geng Y, Lamplugh L, Hekmatnejad B, Gaumond MH, Penney J, Yamanaka Y, Moffatt P: Crispr-Cas9 engineered osteogenesis imperfecta type $\mathrm{V}$ leads to severe skeletal deformities and perinatal lethality in mice. Bone 2018;107:131-142. 